\title{
Diphoton searches (CMS)
}

\author{
Milena Quittnat ${ }^{1, \star}$ on behalf of the CMS collaboration \\ ${ }^{1}$ ETH Zurich
}

\begin{abstract}
The search for high mass resonances decaying into two photons is well motivated by many physics scenarios beyond the standard model. This note summarizes the results of this search in proton-proton collision with a center-of-mass energy of $\sqrt{s}=13 \mathrm{TeV}$ and an integrated luminosity of $3.3 \mathrm{fb}^{-1}$ by the CMS experiment. It presents the interpretation of the results under spin- 0 and spin- 2 hypotheses with a relative width up to $5.6 \times 10^{-2}$ in a mass region of $500-4500 \mathrm{GeV}$. The results of the $\sqrt{s}=13 \mathrm{TeV}$ analysis are combined statistically with previous searches performed by the CMS collaboration, employing a center-of-mass energy of $\sqrt{s}=8 \mathrm{TeV}$ and an integrated luminosity of $19.7 \mathrm{fb}^{-1}$.
\end{abstract}

\section{Introduction}

Several extensions of the standard model (SM) of particle physics predict the production of a resonance decaying into a high mass photon pair. The Landau-Yang theorem postulates a spin of 0 or an integer value of higher than 2 for these resonances [1]. The analysis described focuses on two benchmark models:

- the production of a spin-0 resonance via gluon-gluon fusion.

- an extra dimensional model, the Randall-Sundrum model, predicting a spin-2 resonance.

The so called two-Higgs-doublet model (2HDM) predicts the existence of five additional scalar or pseudo-scalar particles in the Higgs sector [2]. The lightest neutral Higgs particle could be identified with the $125 \mathrm{GeV}$ Higgs boson which was discovered by the CMS and ATLAS experiments in $2012[3,4]$. In the alignment limit, one of the additional neutral Higgs particles with mass $m_{S}$ could decay into two photons at a significant rate and be detected in the CMS or ATLAS detector [5].

Additional space-like dimensions provide an explanation for the observed difference between the Planck and electroweak scales by introducing a gravitational field which also propagates through at least one extra dimension. The metric of the Randall-Sundrum (RS) graviton model [6] is that one of a five-dimensional anti-de-Sitter space with one warped extra-dimension. The RS-model postulates two brane worlds. The SM fields exist only on the 'electroweak brane' whereas gravity is also allowed

^e-mail: milenaq@phys.ethz.ch 
to propagate to the second 'gravity brane'. The mediator of the gravitational force, the graviton, might then be observed as a spin-2 resonance with mass $m_{G}$ in the ATLAS or CMS detector and would decay, among others, into diphotons. In 2015, the ATLAS and CMS collaborations could explore a new energy range using proton-proton collisions at a center-of-mass energy of $\sqrt{s}=13 \mathrm{TeV}$. Both collaborations presented results on searches for diphoton resonances in the mass ranges of $200 \mathrm{GeV}-2 \mathrm{TeV}$ $(500 \mathrm{GeV}-5 \mathrm{TeV}$ ) for spin-0 (spin-2) hypotheses at ATLAS [7] and $500 \mathrm{GeV}-4.5 \mathrm{TeV}$ at CMS [8]. This note summarizes the results from the CMS collaboration at $\sqrt{s}=13 \mathrm{TeV}$ with an integrated luminosity of $3.3 \mathrm{fb}^{-1}$ and reports further on the statistical combination of this search with similar searches by the CMS collaboration performed at $\sqrt{s}=8 \mathrm{TeV}$. The note is based on the results reported in Ref. [9].

\section{Diphoton reconstruction with the CMS detector}

A detailed description of the CMS apparatus, together with the relevant kinematic variables, can be found in Ref. [10]. The CMS detector is designed to include the central tracking and calorimetric system within its solenoid, which provides a magnetic field of $\mathrm{B}=3.8 \mathrm{~T}$. The muon detection system surrounds the solenoid and is placed within the steel skeleton of the detector. The central 'barrel' part of the detector is complemented by a forward region at each side, the so-called 'endcaps'. The reconstruction of photon candidates rely mainly one the electromagnetic calorimeter (ECAL) and to some extent on the tracker system. The electromagnetic calorimeter is built from lead-tungstate crystals providing an excellent intrinsic diphoton energy resolution of approximately $0.5 \%$ for masses above $100 \mathrm{GeV}$. Photon candidates are formed by clustering spatially correlated energy deposits in ECAL. They are reconstructed by the particle-flow event algorithm [11, 12], applying an optimized combination of the information of various detector parts. The energy of the photon candidates is evaluated by a multivariate regression technique. For a detailed description of the applied calibrations, corrections and techniques, Ref. [13] may be consulted.

\section{Event selection and reconstruction}

The full list of the used simulation samples of this analysis can be found in Ref. [9]. The $13 \mathrm{TeV}$ datset with an integrated luminosity $\mathcal{L}$ of $3.3 \mathrm{fb}^{-1}$ is recorded with two different magnetic field configurations: $2.7 \mathrm{fb}^{-1}$ with $\mathrm{B}=3.8 \mathrm{~T}$ and $0.6 \mathrm{fb}^{-1}$ with $\mathrm{B}=0 \mathrm{~T}$. The diphoton final state has a clean experimental signature and two well isolated high momentum photon candidates. The trigger selection thus requires at least two photon candidates of transverse momentum above 60(40) GeV for $\mathrm{B}=3.8 \mathrm{~T}(\mathrm{~B}=0 \mathrm{~T})$ and is fully efficient for the search range of masses $m_{X}$ greater than $0.5 \mathrm{TeV}[9]$. For the final event selection, photon candidates are required to have a transverse momentum of $\mathrm{p}_{\mathrm{T}}>75 \mathrm{GeV}$ and a pseudorapidity of $\left|\eta_{S C}\right|<2.5$ as well as $\left|\eta_{S C}\right|<1.44$ for at least one of them. To maximize the sensitivity of the analysis, the selected events are separated into two categories:

- EBEB: both photons are centrally detected, in the barrel (EB) region of ECAL with $\left|\eta_{S C}\right|<1.44$.

- EBEE: one photon is centrally detected, the other one in the forward region, the endcaps (EE) of ECAL with $1.56<\left|\eta_{S C}\right|<2.5$.

The invariant mass $m_{\gamma \gamma}$ of the diphoton pair is required to be above $230 \mathrm{GeV}(320 \mathrm{GeV})$ for EBEB (EBEE) events. The diphoton mass resolution depends also on the vertex identification. If the difference between the reconstructed vertex and the true vertex is smaller than $1 \mathrm{~cm}$, the effect on the 
mass resolution is negligible. For the $\mathrm{B}=3.8 \mathrm{~T}$ dataset, a boosted decision tree is used for the vertex identification and the interaction vertex is correctly assigned for about $90 \%$ of the signal events [9]. Since this approach relies on the $\mathrm{p}_{\mathrm{T}}$ of the tracks, a different identification method has to be used for $\mathrm{B}=0 \mathrm{~T}$ and subsequently the vertex with the highest track multiplication is chosen. The chosen vertex algorithm improves the expected mass resolution of a possible resonance by approximately $20 \%$ in comparison to the average vertex position as depicted in Figure 1. The probability for the correct vertex assignment is about $60 \%$.

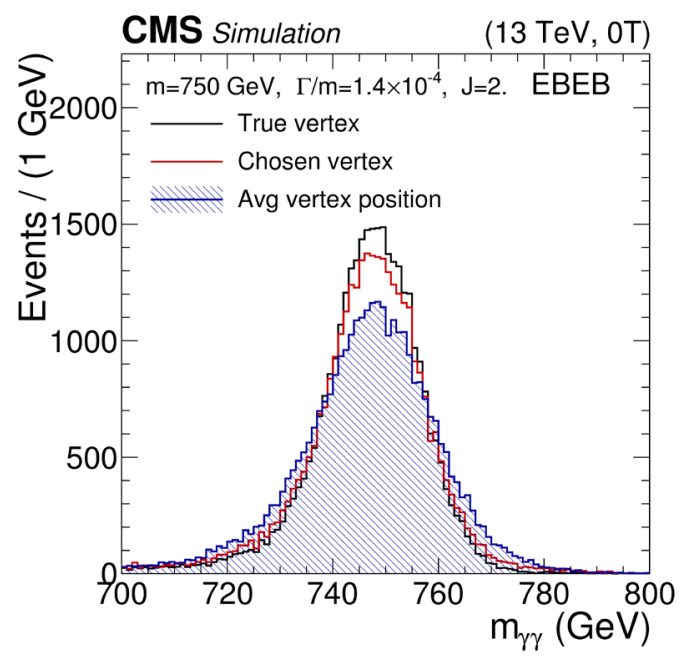

Figure 1. Expected reconstructed invariant mass distribution for a narrow width diphoton resonance at a mass of $m=750 \mathrm{GeV}$ with $\mathrm{B}=0 \mathrm{~T}$ for different primary vertex identifications, when both photons are centrally detected (EBEB).

For the final event selection, the photon candidates are required to satisfy a set of identification criteria. The respective variables and thresholds depend on the value of the magnetic field and are detailed in Ref. [9]. The per-photon identification efficiency is above 90 (85)\% in the barrel (endcaps) for $\mathrm{B}=3.8 \mathrm{~T}$. In the $\mathrm{B}=0 \mathrm{~T}$ dataset, the per-photon identification efficiency is above $85(70) \%$ for prompt isolated photon candidates in the barrel (endcaps), mainly due to a less efficient electron-veto. The overall signal selection efficiency as a function of $m_{X}$ is shown in Figure 2 and varies between $0.5-0.7(0.4-0.5)$ for $\mathrm{B}=3.8 \mathrm{~T}(\mathrm{~B}=0 \mathrm{~T})$, depending on the signal hypothesis. The kinematic acceptance for the RS graviton resonances is approximately $20 \%$ lower than for scalar resonances for masses below $1 \mathrm{TeV}$ due to the different angular distribution of the decay products. The two acceptances become similar for $m_{X}>3 \mathrm{TeV}$. There is nearly no difference in the expected sensitivity for the spin- 0 or spin- 2 hypothesis.

A data-driven tag and probe method is employed to verify the photon selection efficiency using $\mathrm{Z} \rightarrow \mathrm{e}^{+} \mathrm{e}^{-}$events. The electrons have to satisify the selection for the high momentum photon candidates with an inverted electron veto. The photon selection efficiency in data and the ratio to the Monte-Carlo (MC) prediction as a function of the transverse momentum $p_{T}$ is shown in Figure 3. The scale factor is compatible with 1 in almost all $p_{T}$-bins and flat within a few percents. A 8 (16) \% un- 

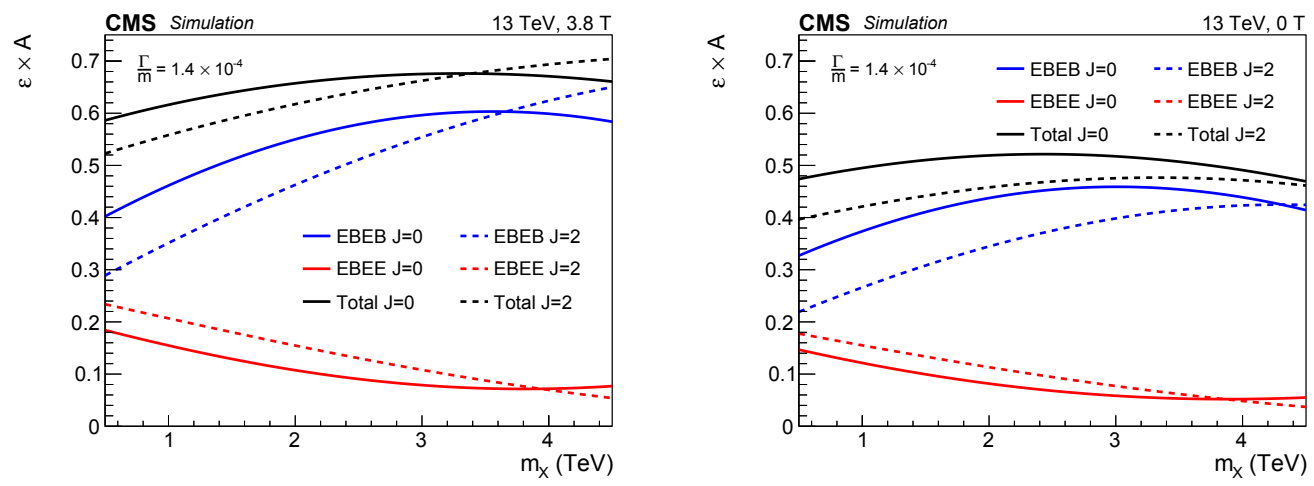

Figure 2. Fraction of events selected by the analysis categories for a possible signal of $500 \mathrm{GeV}<m_{X}<4.5 \mathrm{TeV}$ and $\Gamma_{X} / m_{X}=1.4 \times 10^{-4}$. The prediction for both, scalar and RS graviton resonances, are shown, on the left for the $\mathrm{B}=3.8 \mathrm{~T}$ sample and on the right for the $\mathrm{B}=0 \mathrm{~T}$ one.

certainty on the selection efficiency is propagated to the signal normalization at $\mathrm{B}=3.8 \mathrm{~T}(\mathrm{~B}=0 \mathrm{~T})$.
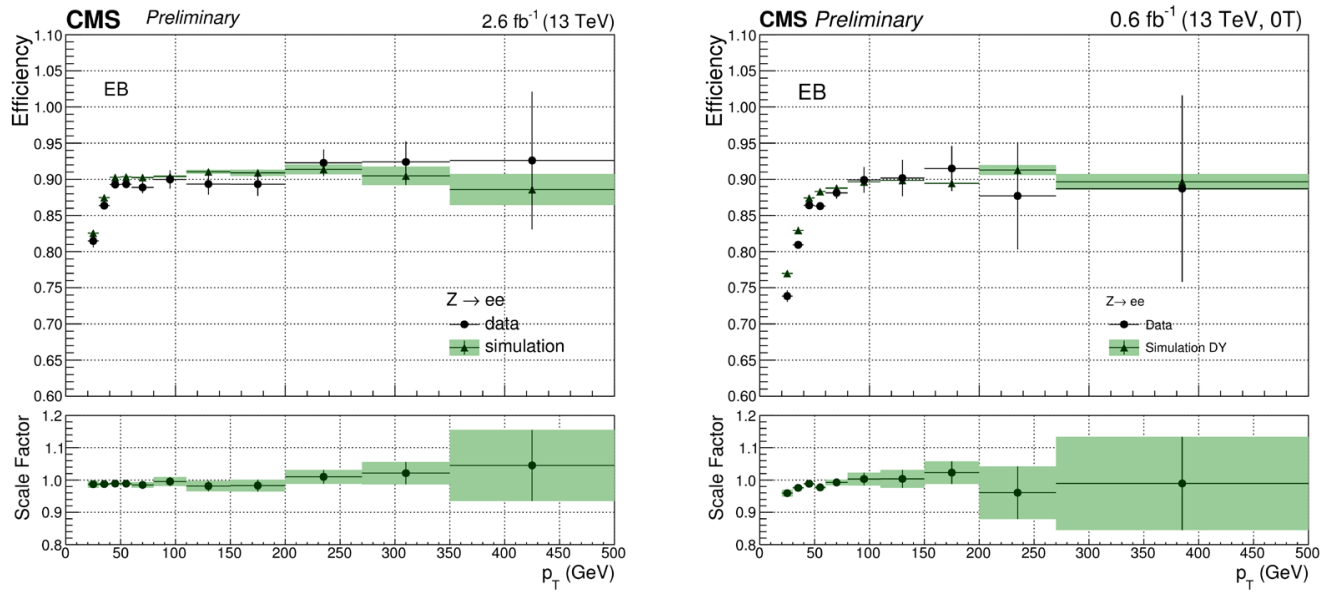

Figure 3. Photon selection efficiency measured with $\mathrm{B}=3.8 \mathrm{~T}$ (left) and $\mathrm{B}=0 \mathrm{~T}$ (right) using the tag and probe method (all cuts are applied except for electron rejection) for the EB category. Bottom: data over Monte-Carlo scale factors.

\subsection{Energy corrections}

The reconstruction and energy assignment of photon candidates is generally derived for lower energy photons than the ones entering this analysis. Thus, the energy scale and energy resolution might 
need to be corrected for higher values. As the energy determination is only dependent on ECAL, well-known electron objects from $\mathrm{Z} \rightarrow \mathrm{e}^{+} \mathrm{e}^{-}$events can be used instead of photon objects. Residual differences in the observed and predicted distributions of a $\mathrm{Z} \rightarrow \mathrm{e}^{+} \mathrm{e}^{-}$sample are thus determined by a tag and probe method with a procedure described in Ref. [13]. The energy scale corrections are about $0.5(1.5) \%$ for photon candidate compatibles at $\mathrm{B}=3.8 \mathrm{~T}(\mathrm{~B}=0 \mathrm{~T})$. The additional Gaussian smearing for the MC prediction is similar for both values of the magnetic field and of the order of $0.8 \%$ and $1.5 \%(2-2.5 \%)$ for photon candidates which are centrally (in the forward region) detected. The energy scale correction factors measured for the $\mathrm{B}=0 \mathrm{~T}$ dataset are found to be about $1 \%$ higher than the $\mathrm{B}=3.8 \mathrm{~T}$ factors. The comparison with the $\mathrm{MC}$ prediction after all energy corrections are applied is reported in Figure 4. The variation of the corrections is studied with $\mathrm{Z} \rightarrow \mathrm{e}^{+} \mathrm{e}^{-}$events as a function of the transverse momentum $\mathrm{p}_{\mathrm{T}}$ up to $150(100 \mathrm{GeV})$ for $\mathrm{EB}(\mathrm{EE})$. The variation is smaller than $0.5(0.7) \%$ for EB (EE) in both datasets.
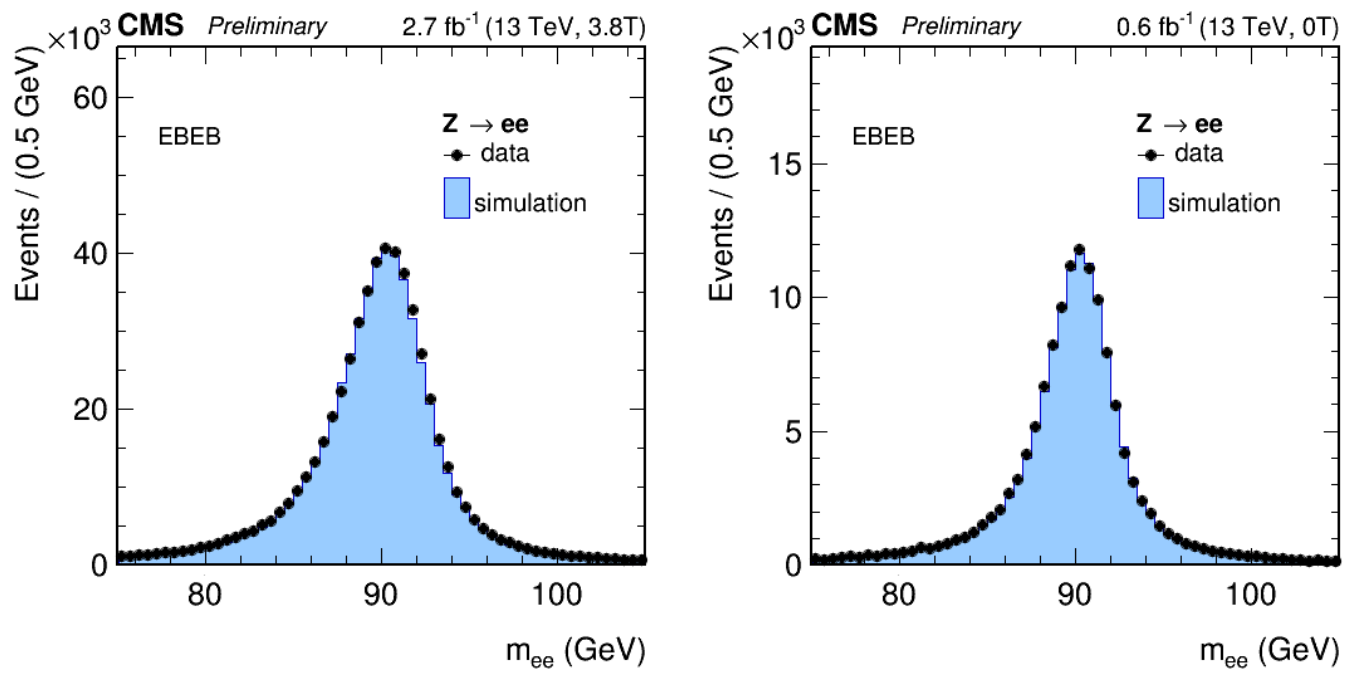

Figure 4. Comparison between the predicted and observed invariant mass distribution of electron pairs obtained after the application of energy scale and resolution corrections. Distributions are shown for events where both electrons are reconstructed for $\mathrm{B}=3.8 \mathrm{~T}$ (left) and $\mathrm{B}=0 \mathrm{~T}$ (right) in the EBEB category. The simulation predictions are scaled to match the number of events observed in data.

\section{Diphoton mass spectrum}

The diphoton spectrum of the events entering the analysis is shown in Figure 5 for all four categories $((\mathrm{B}=3.8 \mathrm{~T}, \mathrm{~B}=0 \mathrm{~T}) \times(\mathrm{EBEB}, \mathrm{EBEE}))$.

The individual background components of the diphoton sample at $\mathrm{B}=3.8 \mathrm{~T}$ are estimated by a datadriven method. This evaluation measures that about $90(80) \%$ of the background events in the EBEB (EBEE) category originate from the irreducible $\gamma \gamma$-SM background processes. This estimate is compared to a precise next-to-next-to leading-order (NNLO) prediction of the $\gamma \gamma$-SM background processes. It is obtained by calculating the so-called 'k-factor', the ratio of the theoretical calculation of 

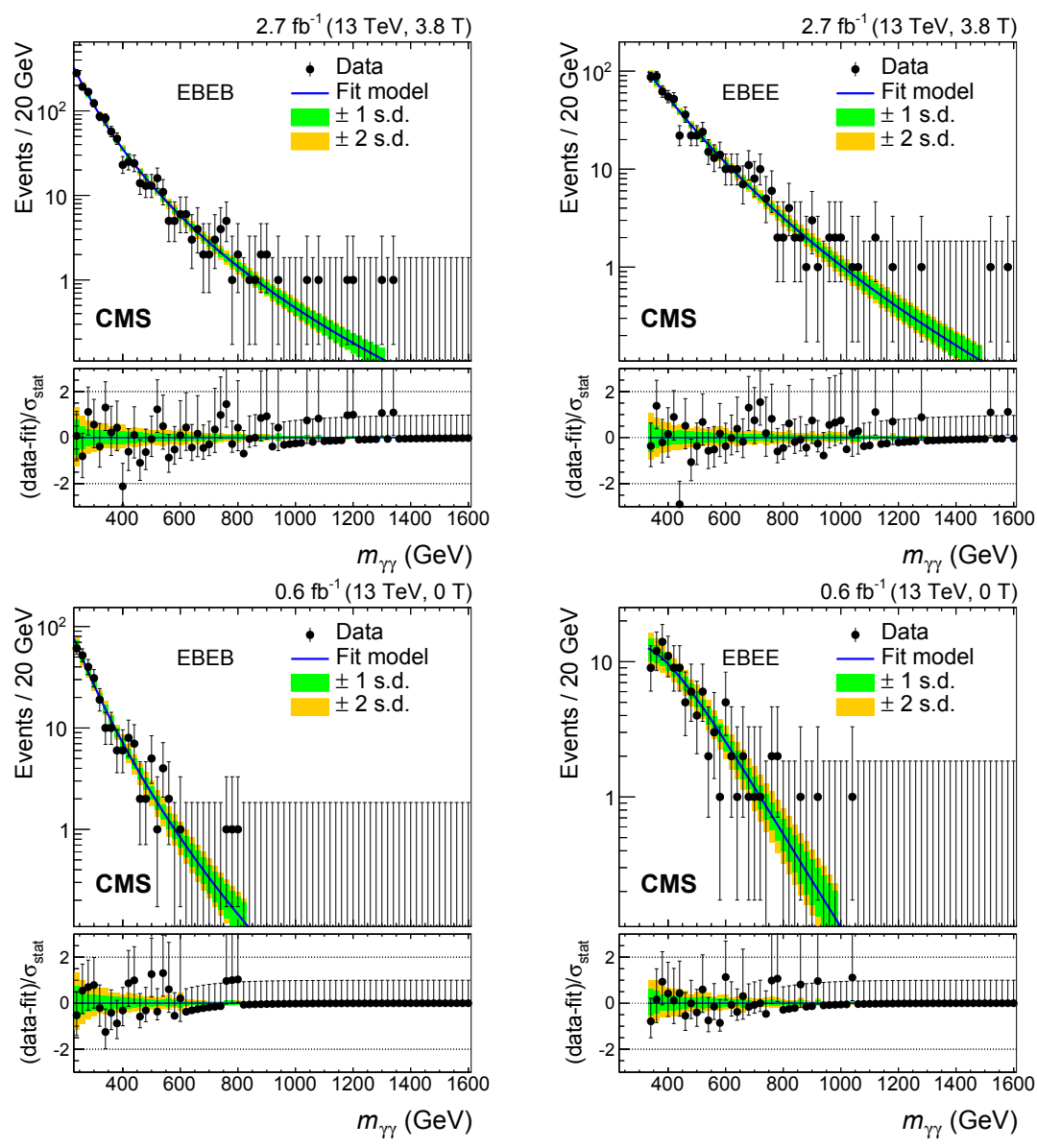

Figure 5. The observed invariant mass spectra are shown for the EBEB (left) and EBEE category (right). The top (bottom) row shows the results for the $\mathrm{B}=3.8 \mathrm{~T}(\mathrm{~B}=0 \mathrm{~T})$ dataset. The results of the parametric fits to the data are depicted with their uncertainties.

the $2 \mathrm{gNNLO}$ [14] simulation program with the leading-order SHERPA simulation at gen-level. The leading-order $\gamma \gamma$-prediction, a SHERPA event generator sample emulated with the detector response, is then reweighed with this k-factor. The comparison of the $\gamma \gamma$ spectrum with the NNLO MC prediction is reported in Figure 6. The predictions from perturbative QCD match the observations within their uncertainties.

\section{Statistical analysis}

A simultaneous unbinned likelihood fit to $m_{\gamma \gamma}$ evaluates the diphoton distributions in all four analysis categories $((\mathrm{B}=3.8 \mathrm{~T}, \mathrm{~B}=0 \mathrm{~T}) \times(\mathrm{EBEB}, \mathrm{EBEE}))$ and for each signal hypothesis $S$ :

$$
\left.L(\mu, \theta)=\Pi_{i=1}^{N_{\text {events }}}\left[\mu \cdot S\left(m_{i} \mid \theta_{S}\right)\right]+B\left(m_{i} \mid \theta_{B}\right)\right] \cdot \operatorname{Pois\operatorname {son}}\left(N_{\text {events }} \mid N_{B}+\mu \cdot N_{S}\right)
$$



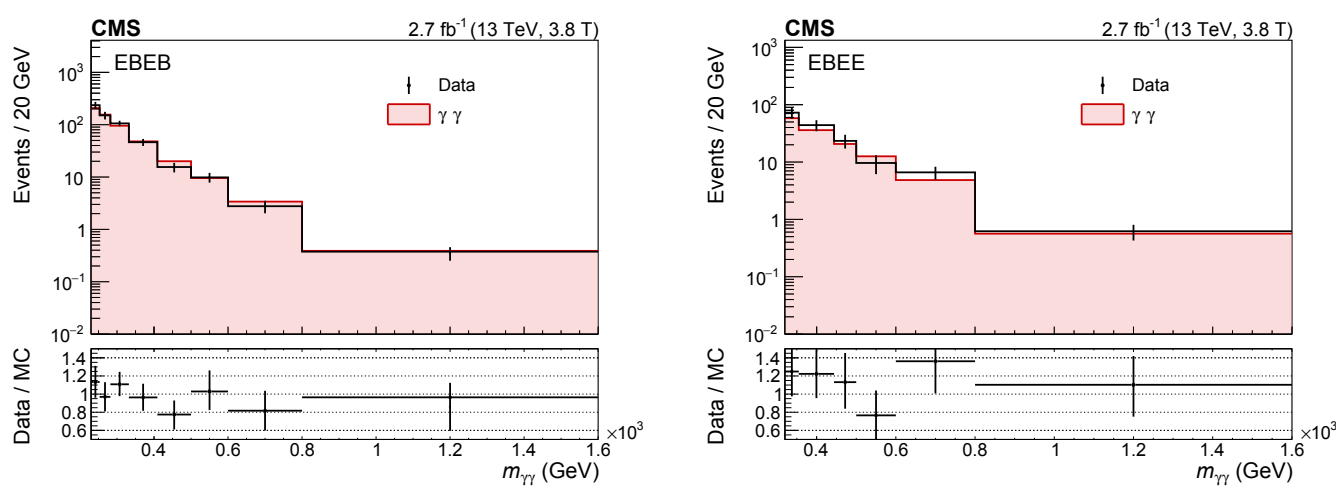

Figure 6. Comparison between the predicted and measured $\gamma \gamma$ background spectrum. Events in the EBEB (EBEE) category are shown on the left (right).

where $B$ denotes the SM background model. The signal and background model depend on the mass $m_{i}$ of event $i$ and their respective nuisance parameters $\theta$, which model the systematic uncertainties. The parameter $\mu$ denotes the signal strength of the considered model. The interpretation is performed with a composite statistical hypothesis test. The test statistics are based on a profile likelihood ratio of the form:

$$
q(\mu)=-\log \lambda(\mu):=-\log \frac{L\left(\mu \cdot S+B \mid \underline{\hat{\theta}}_{\mu}\right)}{L(\hat{\mu} \cdot S+B \mid \underline{\hat{\theta}})}
$$

relying on the likelihood $L$. The $\hat{x}$ notation denotes the best fit value of parameter $x$, the notation $\hat{x}_{y}$ symbolizes the best fit value of $x$, conditionally on $y$.

The shape of the diphoton distribution as a function of $m_{\gamma \gamma}$ models the background expectation using the functional form:

$$
g\left(m_{\gamma \gamma}\right)=m_{\gamma \gamma}^{a+b \cdot \log \left(m_{\gamma \gamma}\right)}
$$

The coefficients are obtained through the maximum likelihood fit to data. Independent coefficients are used for each analysis category and are considered as unconstrained nuisance parameters. The uncertainty on the accuracy of the background determination is assessed and accounted for in the hypothesis test [9].

The signal distribution as a function of $m_{\gamma \gamma}$ is described as the convolution of the intrinsic shape of the resonance and the ECAL detector response [9, 19]. The signal hypotheses considered herein are simulated at leading order with the PYTHIA [20] event generator, using NNPDF 2.3 [21] parton distribution functions. Three different values of relative width are used as benchmark models:

- $\Gamma_{X}$ is much smaller than the detector resolution: $\Gamma_{X} / m_{X}=1.4 \times 10^{-4}$.

- $\Gamma_{X}$ is of the same size as the detector resolution: $\Gamma_{X} / m_{X}=1.4 \times 10^{-2}$.

- $\Gamma_{X}$ much larger than the detector resolution: $\Gamma_{X} / m_{X}=5.6 \times 10^{-2}$.

For the RS graviton model, the relative widths correspond to the dimensionless coupling parameter

$$
\tilde{k}=\sqrt{\frac{\Gamma_{X} / m_{X}}{1.4}}=0.01,0.1, \text { and } 0.2
$$


as described in Ref. [22]. In order to determine the signal normalization, the efficiency of the final event selection is combined with the kinematic acceptance as shown in Figure 2. In this analysis, the statistical uncertainties dominate over the systematic ones. In addition to the above mentioned systematics on the background model, the selection efficiency and the photon energy scale, the luminosity uncertainty and the uncertainty on the parton distribution function enter the hypothesis test.

For the interpretation of the results, frequentist statistics and asymptotic formulas [15] are used throughout and their validity is tested [9]. Thus, upper limits on the resonant diphoton production rate under different signal hypotheses are evaluated with the modified frequentist method, also known as $\mathrm{CL}_{\mathrm{s}}[16,17]$. The agreement of the observed result with the background-only hypothesis is estimated by the background-only $p$-value, the 'local $p$-value' $p_{0}[18]$.

\section{Results of the search at $\sqrt{s}=13 \mathrm{TeV}$}

For the $\sqrt{s}=13 \mathrm{TeV}$ dataset, the search range for a possible resonance with mass $m_{X}$ covers the region $500 \mathrm{GeV}<m_{X}<4.5 \mathrm{TeV}$. The median expected and observed 95\% C.L. exclusion limits on the production of scalar and RS graviton resonances are reported in Fig. 7 for the narrow and the largest width hypotheses. The complete list of results for the exlusion limits as well as for the background-only $p$-value can be found in Ref. [9]. In Figure 8, the results for a relative width of $\Gamma_{X} / m_{X}=1.4 \times 10^{-2}$ are shown on the left. This signal hypothesis shows the largest excess in data among all considered widths hypotheses at a mass of $m_{X}=760 \mathrm{GeV}$. The background-only p-value for the RS graviton model is depicted on the right side of that figure and shows a local significance of 2.9 standard deviations. The separate $\mathrm{B}=3.8 \mathrm{~T}$ and $\mathrm{B}=0 \mathrm{~T}$ contributions point out that the $\mathrm{B}=0 \mathrm{~T}$ category adds approximately $20 \%$ to the observed excess due to its lower luminosity. The probability of observing at least one excess more significant than that in the full search range and among all considered models of this analysis, is the so-called "look-elsewhere-effect" [23]. It is derived from a sampling distribution of $\max \left(p_{0}\right)$ of an ensemble of background-only pseudo datasets and found to be less than one standard deviation.
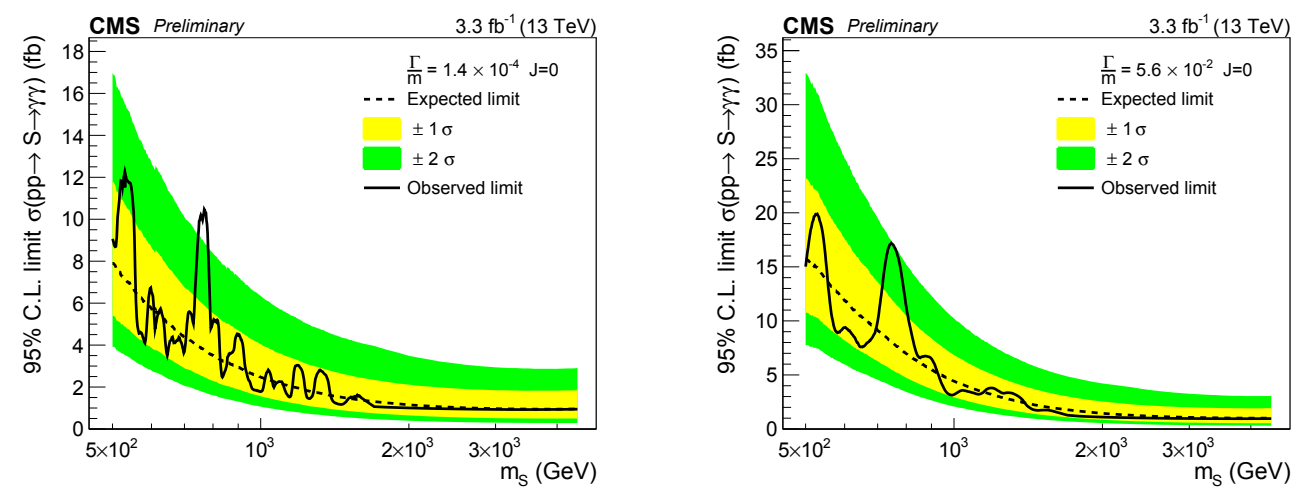

Figure 7. Expected and observed 95\% C.L. exclusion limits in the range $500 \mathrm{GeV}<m_{X}<4.5 \mathrm{TeV}$ for $\Gamma_{X} / m_{X}=1.4 \times 10^{-4}$ (left) and $5.6 \times 10^{-2}$ (right) for the scalar $(\mathrm{J}=0)$ resonance hypothesis. 

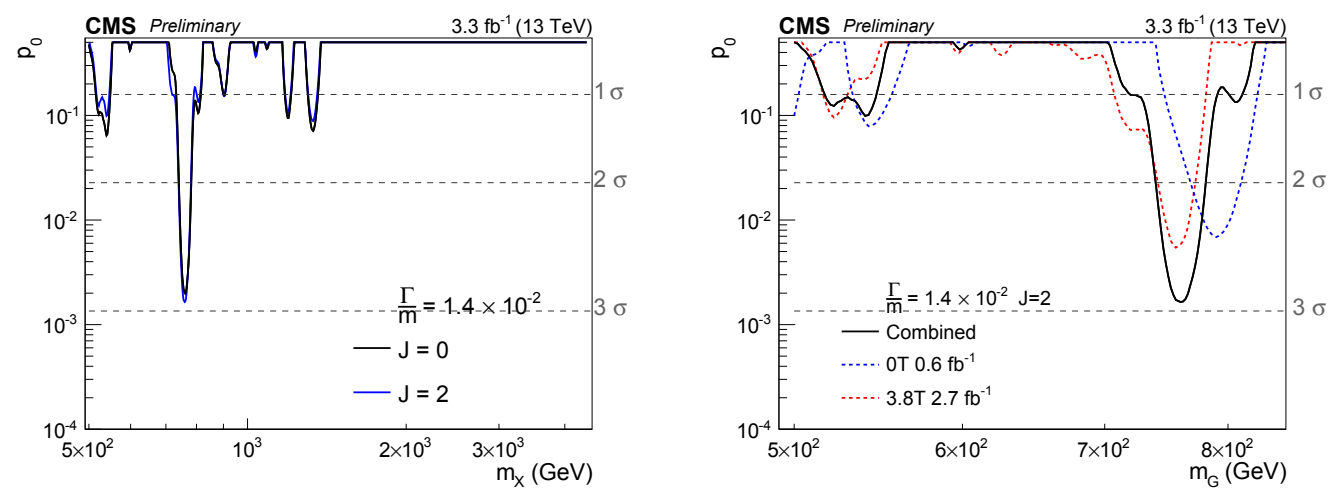

Figure 8. Observed background only p-values obtained on the $13 \mathrm{TeV}$,dataset. For $\Gamma_{X} / m_{X}=1.4 \times 10^{-2}$, the range $500 \mathrm{GeV}<m_{X}<4.5 \mathrm{TeV}(850 \mathrm{GeV})$ is shown on the left (right). Results corresponding to both spin hypotheses are shown on the left. The contributions of the $\mathrm{B}=3.8 \mathrm{~T}$ and $\mathrm{B}=0 \mathrm{~T}$ datasets are shown separately on the right for the spin-2 hypothesis. Due to the different integrated luminosity, the weight of the $\mathrm{B}=0 \mathrm{~T}$ dataset at $m_{X}=760 \mathrm{GeV}$ is approximately $20 \%$ of the $\mathrm{B}=3.8 \mathrm{~T}$ one in the combined result.

\section{Results of the combined analysis of $\sqrt{s}=8 \mathrm{TeV}$ and $13 \mathrm{TeV}$ datasets}

The results of the $\sqrt{s}=13 \mathrm{TeV}$ dataset are combined statistically with results obtained by the CMS collaboration at a center-of-mass energy of $\sqrt{s}=8 \mathrm{TeV}$ employing an integrated luminosity of $19.7 \mathrm{fb}^{-1}$. Two searches for resonant diphoton production were performed with the $8 \mathrm{TeV}$ dataset:

- an analysis searching for spin-0 and spin-2 resonances in the mass range $150-850 \mathrm{GeV}$ [24].

- a second analysis focusing on spin-2 resonances with a search range of $500 \mathrm{GeV}-3 \mathrm{TeV}$ [25].

The two event samples partially overlap, thus at each $m_{X}$, the analysis with the most stringent median expected exclusion limit on resonant diphoton production is taken for the final combination. For resonance masses below $m_{X}<850 \mathrm{GeV}$, the results of Ref. [24] enter the combination, and those of Ref. [25] otherwise. The ratio of the signal production cross sections at $8 \mathrm{TeV}$ and $13 \mathrm{TeV}$ has been calculated [9]. It is approximately

- $0.29(0.27)$ for $m_{G}\left(m_{S}\right)=500 \mathrm{GeV}$,

- $0.24(0.22)$ for $m_{G}\left(m_{S}\right)=750 \mathrm{GeV}$,

- $0.04(0.03)$ for masses in the TeV range $\left(m_{G}\right.$ or $\left.m_{S} \approx 3 \mathrm{TeV}\right)$.

For further details, Ref. [9] may be consulted. The combined result is obtained by a simultaneous fit to the $m_{\gamma \gamma}$ spectra in all event categories, assuming a common signal strength modifier for all categories. The median expected and observed $95 \% \mathrm{CL}_{s}$ exclusion limits on the equivalent $13 \mathrm{TeV}$ diphoton resonance production cross section, $\sigma_{G, S}^{13 \mathrm{TeV}} \cdot \mathcal{B}_{\gamma \gamma}$, for the combined analysis are reported in Figure 9 for all studied signal hypothesis of this analysis. The comparison with Figure 7 shows an improvement of the exclusion limits by $20-40 \%$ for masses below $1.5 \mathrm{TeV}$. For 
masses above $1.5 \mathrm{TeV}$, the exclusion limits are mainly driven by the sensitivity of the $13 \mathrm{TeV}$ analysis and the benefits of the combination decrease. Randall-Sundrum gravitons with masses below approximately 1.6, 3.3, and $3.8 \mathrm{TeV}$ are excluded at a $95 \%$ confidence level for $\tilde{\kappa}=0.01,0.1$, and 0.2 , respectively, corresponding to a relative widths of $\Gamma_{X} / m_{X}=1.4 \times 10^{-4}, 1.4 \times 10^{-2}$ and $5.6 \times 10^{-2}$. Similar exclusion limits are found for the scalar signal hypotheses. The result of the local significance for a scalar resonance under the narrow width hypothesis is depicted in Figure 10. This model shows the largest excess in the combination among all considered signal hypotheses with a local significance of roughly 3.4 standard deviations at a mass of $m_{S}=750 \mathrm{GeV}$. Further results can be found in Ref. [9]. Taking into account the effect of searching for a diphoton resonance among all six signal hypotheses, the global significance is estimated to be approximately 1.6 standard deviations [9].

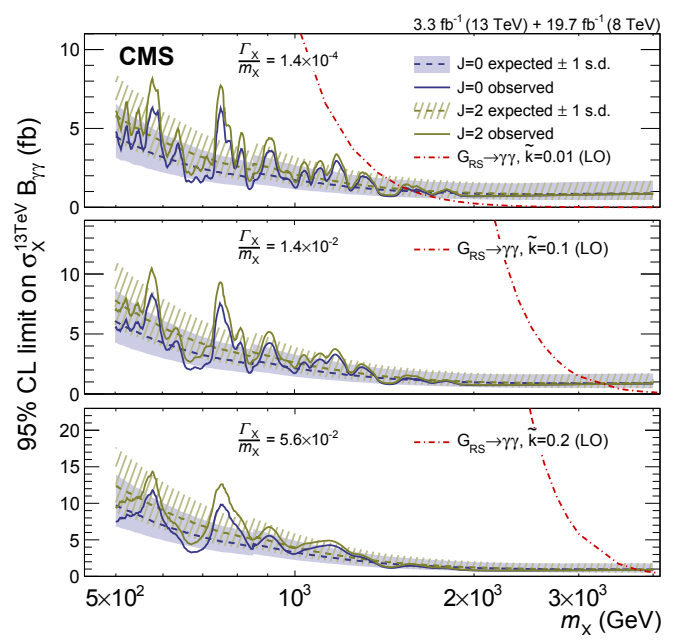

Figure 9. Expected and observed 95\% C.L. exclusion limits in the range $500 \mathrm{GeV}<m_{X}<3 \mathrm{TeV}$ for both spin hypothesis with a relative width of $\Gamma_{X} / m_{X}=1.4 \times 10^{-4}$ (top), $\Gamma_{X} / m_{X}=1.4 \times 10^{-2}$ (middle) and $\Gamma_{X} / m_{X}=5.6 \times$ $10^{-2}$ (bottom).

\section{Summary and outlook}

This note summarizes the search for high mass resonances decaying into the diphoton final state by the CMS experiment [9]. The analysis employs a dataset of $3.3 \mathrm{fb}^{-1}$ and $19.7 \mathrm{fb}^{-1}$ of protonproton collisions at a center-of-mass energy of $\sqrt{s}=13 \mathrm{TeV}$ and $\sqrt{s}=8 \mathrm{TeV}$, respectively. The search sets $95 \% \mathrm{CL}_{\mathrm{s}}$ exclusion limits on the resonant diphoton production of scalar resonances $(\mathrm{J}=0)$ and RS gravitons $(\mathrm{J}=2)$ in the mass range of $500 \mathrm{GeV}<m_{X}<4.5 \mathrm{TeV}$ for relative width up to $\Gamma_{X} / m_{X}=5.6 \times 10^{-2}$, using leading-order simulation for the signal processes. Randall-Sundrum gravitons with masses below approximately $1.6,3.3$, and $3.8 \mathrm{TeV}$ are excluded at a $95 \%$ confidence level for $\tilde{\kappa}=0.01,0.1$, and 0.2 , respectively, corresponding to a relative widths of $\Gamma_{X} / m_{X}=1.4 \times$ $10^{-4}, 1.4 \times 10^{-2}$ and $5.6 \times 10^{-2}$. A modest excess of events over the background-only hypothesis is observed for $m_{X}=750 \mathrm{GeV}$. The corresponding local significance under the narrow-width hypothesis $\left(\Gamma_{X} / m_{X}=1.4 \times 10^{-4}\right)$ for a spin- 0 particle is approximately 3.4 standard deviations. Taking into 

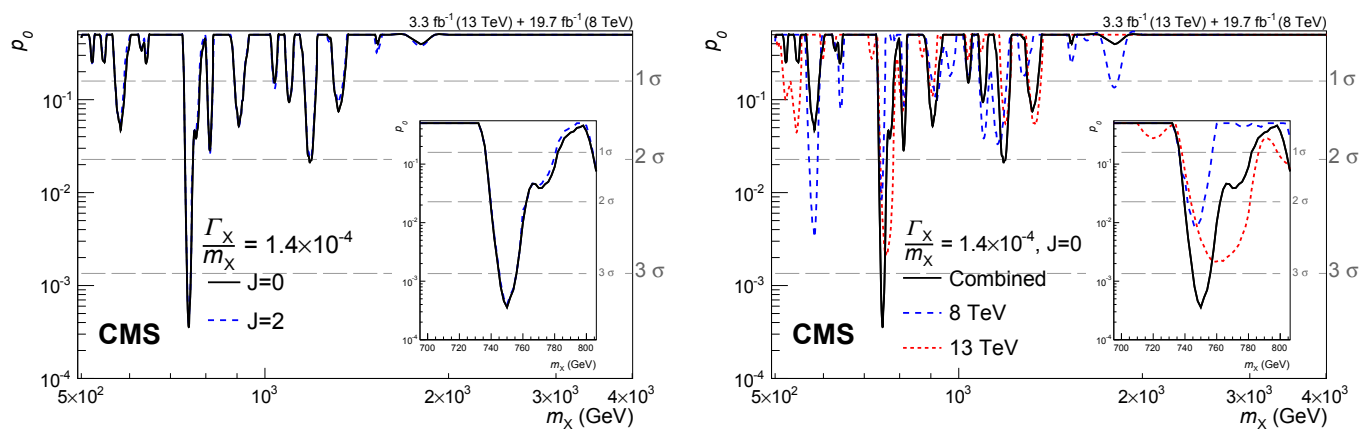

Figure 10. Left: observed background-only p-values obtained on the 8 and $13 \mathrm{TeV}$ combination for both spin hypothesis and a relative width of $\Gamma_{X} / m_{X}=1.4 \times 10^{-4}$. Right: observed background-only p-values for the spin- 0 hypothesis with a relative width of $\Gamma_{X} / m_{X}=1.4 \times 10^{-4}$. The contributions of the 8 and $13 \mathrm{TeV}$ datasets are shown separately.

account all signal hypotheses considered in the analysis, the global significance of this excess is estimated to 1.6 standard deviations. 


\section{References}

[1] L.D. Landau, Dokl. Akad. Nauk SSSR 60, 207 (1948)

[2] N. Craig, J. Galloway, S. Thomas (2013), 1305.2424

[3] G. Aad et al. (ATLAS), Phys. Lett. B716, 1 (2012), 1207. 7214

[4] S. Chatrchyan et al. (CMS), Phys. Lett. B716, 30 (2012), 1207.7235

[5] P.S.B. Dev, A. Pilaftsis (2014), 1408. 3405

[6] L. Randall, R. Sundrum, Phys. Rev. Lett. 83, 3370 (1999), hep-ph/9905221

[7] Journal of High Energy Physics 2016, 1 (2016)

[8] CMS Collaboration (CMS), CMS Physics Analysis Summary CMS-PAS-EXO-15-004 (2015), https://cds. cern.ch/record/2114808

[9] V. Khachatryan et al. (CMS Collaboration), Phys. Rev. Lett. 117, 051802 (2016)

[10] S. Chatrchyan et al. (CMS), JINST 3, S08004 (2008)

[11] CMS Collaboration (CMS), CMS Physics Analysis Summary CMS-PAS-PFT-09-001 (2009), http: //cdsweb.cern.ch/record/1194487

[12] CMS Collaboration (CMS), CMS Physics Analysis Summary CMS-PAS-PFT-10-001 (2010), http://cdsweb. cern. ch/record/1247373

[13] V. Khachatryan et al. (CMS), JINST 10, P08010 (2015), 1502.02702

[14] S. Catani, L. Cieri, D. de Florian, G. Ferrera, M. Grazzini, Phys.Rev.Lett. 108, 072001 (2012), 1110.2375

[15] G. Cowan, K. Cranmer, E. Gross, O. Vitells, Eur.Phys.J. C71, 1554 (2011), 1007. 1727

[16] T. Junk, Nucl. Instrum. Meth. A 434, 435 (1999), hep-ex/9902006

[17] A. Read, Tech. Rep. CERN-OPEN-2000-005, CERN (2000), http: //cdsweb. cern.ch/record/451614

[18] ATLAS and CMS Collaborations, LHC Higgs Combination Group, ATL-PHYS-PUB/CMS NOTE 2011-11, 2011/005 (2011), http: //cdsweb . cern . ch/record/1379837

[19] M. Baak, S. Gadatsch, R. Harrington, W. Verkerke, Nucl. Instrum. Meth. A 771, 39 (2015), 1410.7388

[20] T. Sjöstrand, S. Ask, J.R. Christiansen, R. Corke, N. Desai, P. Ilten, S. Mrenna, S. Prestel, C.O. Rasmussen, P.Z. Skands, Comput. Phys. Commun. 191, 159 (2015), 1410.3012

[21] R.D. Ball et al., Nucl. Phys. B867, 244 (2013), 1207.1303

[22] H. Davoudiasl, J.L. Hewett, T.G. Rizzo, Phys. Rev. Lett. 84, 2080 (2000), hep-ph/9909255

[23] E. Gross, O. Vitells, Eur. Phys. J. C70, 525 (2010), 1005. 1891

[24] V. Khachatryan et al. (CMS), Phys. Lett. B750, 494 (2015), 1506.02301

[25] CMS Physics Analysis Summary CMS-PAS-EXO-12-045, CERN (2015), http ://cds . cern. ch/record/2017806 